Original Paper http://ajol.info/index.php/ijbcs http://indexmedicus.afro.who.int

\title{
Amélioration du sol dégradé par l'apport d'engrais inorganique, organiques et évaluation de rendement du maïs (Zea mays L.) dans la province de Lomami, République Démocratique du Congo
}

\author{
Remy Tshibingu MUKENDI ${ }^{1 *}$, Benjamin Tatangolo MUTAMBA ${ }^{1}$, \\ Dominique Musenge $\mathrm{KABONGO}^{2}$, Louis Baboy LONGANZA ${ }^{3}$ \\ et Theodore Mushambani MUNYULI ${ }^{4}$ \\ ${ }^{1}$ Département de Phytotechnie, Faculté des Sciences Agronomiques, Université Notre Dame de Lomami ; \\ Province de Lomami, République Démocratique du Congo. \\ ${ }^{2}$ Département de Sciences, Institut Supérieur et Pédagogique de Kabinda, Province de Lomami, République \\ Démocratique du Congo. \\ ${ }^{3}$ Département de phytotechnie, Faculté des Sciences Agronomiques, Université de Lubumbashi, \\ Collaborateur Scientifique à l'Université Libre de Bruxelles, Service d'Écologie du Paysage et Systèmes de \\ Production Végétale, Avenue F.D. Roosevelt 50, CP 169 B-1050 Bruxelles, Belgique. \\ ${ }^{4}$ Centre National de Recherche en Sciences Naturelles (CRSN/Lwiro) et Université du Cinquantenaire de \\ Lwiro (UNIC/Lwiro), D.S. Bukavu, Province du Sud-Kivu, République Démocratique du Congo. \\ *Auteur correspondant; E-mail : mukendiremy@gmail.com; Tél : (243) 995070206
}

\section{RESUME}

L'étude est effectuée à Kabinda sur un sol dégradé ayant reçu les apports des engrais organiques et minéral dans l'optique d'évaluer leurs effets sur la croissance et la production du maïs. L'expérimentation, conduite pendant deux campagnes agricoles, la saison A et la saison B, était faite suivant un dispositif en blocs complets randomisés (RCBD). Les traitements en quatre répétitions ont été constitués d'un témoin (T0), de quatre niveaux d'apport de fertilisant: d'Entada abyssinica (T1), Tithonia diversifolia (T2) Sena floribunda (T3), et du Diammonophosphate DAP (T4). Les observations avaient porté sur les paramètres de croissance et de production qui ont subit l'analyse de la variance (ANOVA) au seuil de 5\%. Les résultats obtenus renseignent que le maïs a répondu positivement dans la phase de croissance sous effet de fertilisant indistinctement de leur nature $(\mathrm{p}<0.05)$ par rapport au témoin dans les deux saisons de culture. L'apport des fertilisants ayant accéléré la croissance végétative d'une part, et a permis d'augmenter le rendement de 40 à $80 \%$ du maïs comparativement au témoin indistinctement de la saison culturale soit $1398.1 \mathrm{~kg} / \mathrm{ha}$ avec $T$. diversifolia et $885.5 \mathrm{~kg} /$ ha pour le témoin en saison A alors qu'en saison B, $3332.8 \mathrm{~kg} /$ ha produite sous DAP et $1825 \mathrm{~kg} / \mathrm{ha}$ en parcelle témoin d'autre part. Ces fertilisants organiques à base de d'E. abyssinica, $T$. diversifolia, S. floribunda ont retenu l'attention pour leurs meilleurs scores en rendement en saison A et avec un écart non significatif du traitement inorganique en saison B. Ces espèces peuvent être considérées comme des sources non négligeables de fertilisant des sols dégradés pour l'amélioration du rendement de maïs. (C) 2017 International Formulae Group. All rights reserved.

Mots clés : Tithonia diversifolia, Sena floribunda, Entada abyssinica, Diammonophosphate (DAP), maïs, rendement. 


\title{
Improvement of soil degraded by inorganic fertilizer, organic matter and yield evaluation of maize (Zea mays L.) in Lomami province, Democratic Republic of Congo
}

\begin{abstract}
The study was carried out in Kabinda on a degraded soil that has received inputs from organic and mineral fertilizers in order to assess their effects on maize growth and production. The experiment was conducted during two crop years, season A and season B. Five treatments in four replicates consisted of one control (T0), four levels of fertilizer (inorganic and organic matter) supply: Entada abyssinica (T1), Tithonia diversifolia (T2) Sena floribunda (T3), and Diammonophosphate DAP (T4) were evaluated using a randomized complete block system (RCBD). The observations had related to the parameters of growth and production that underwent the analysis of variance (ANOVA) at $5 \%$ level of significance. The results obtained indicate that maize responded positively in the growth stage under the effect of fertilizing indiscriminately of their nature $(p$ $<0.05)$ compared with the control in the two growing seasons. The fertilizers accelerated vegetative growth and increased the yield from 40 to $80 \%$ of maize compared with the control of the cropping season indiscriminately. T. diversifolia gave higher seed yield $1398.1 \mathrm{~kg} /$ ha and control had lower seed yield 885.5 $\mathrm{kg} /$ ha in season A whereas in season B, DAP produced higher seed yield $3332.8 \mathrm{~kg} /$ ha and the control plot gave lower seed yield 1825.kg / ha. These organic fertilizers based on E. Abyssinica, T. diversifolia, and $S$. floribunda were noted for their high yield scores in season A and a non-significant difference in inorganic treatment in season B. These species can be considered as significant sources of soil fertilizer to improve maize yield.

(C) 2017 International Formulae Group. All rights reserved.
\end{abstract}

Keywords: Tithonia diversifolia, Sena floribunda, Entada abyssinica, Diammonophosphate (DAP), maize, yield.

\section{INTRODUCTION}

Le maïs est la troisième céréale cultivée dans le monde après le blé et le riz. Il occupe la première place en République Démocratique du Congo et est cultivé comme culture vivrière de premier ordre. La forte demande de cette denrée est faite en province du Katanga, de trois Kasai et de Lomami en particulier en RD Congo (Mukendi et al., 2017)

En alimentation humaine, ses grains sont transformés en farine pour la préparation de la pâte alimentaire appelée localement shima et biashi par la population de Lomami. Ils sont en plus transformés en une liqueur traditionnelle communément appelée lotoko ou tshitshampa, bue dans les rites et cérémonies locales comme le mariage, le deuil et autres divertissements. Ses épis sont consommés frais, bouillis, braisés ou grillés.

Cependant, malgré son importance alimentaire, son rendement moyen au niveau national dans les exploitations paysannes est très faible $(0,8$ à $1 \mathrm{t} / \mathrm{ha})$ comparativement à d'autres pays comme l'Italie (9530 kg/ha), Canada (6630 kg/ha), Chine (4570 kg/ha), l'Argentine $(5650 \mathrm{~kg} / \mathrm{ha})$, etc. (Tahir et al., 2009). Dans la zone d'étude, Province de Lomami, son rendement en culture traditionnelle est encore largement inférieur à $800 \mathrm{~kg} / \mathrm{ha}$ (Mukendi et al., 2017). Les raisons majeures de cette faible production sont nombreuses dont entre autres la faible maîtrise de nouvelles méthodes de productions agricoles et l'usage des pratiques de production non soutenable (Namoi et al., 2014) mais aussi le déclin de fertilité du sol 
(Ngome et al., 2012). Savini et al. (2006) pensent qu'en Afrique $88 \%$ des sols sont déficients en phosphore, ce qui permet l'obtention de rendement faible. Bationo et al. (2006) ont souligné que la faible fertilité des sols est reconnue de plus en plus comme la principale cause biophysique de la diminution de la productivité des sols des régions d'Afrique subsaharienne avec comme conséquence des rendements très bas de cultures de plus en plus décriée de partout le monde.

Pourtant, en système traditionnel, les paysans préconisent d'ordinaire l'assolement et dépendent étroitement de la nature du sol. Mais, suite à la demande croissante du maïs qui constitue une denrée alimentaire et source de revenus de plus en plus importante pour la plupart des exploitants agricoles. L'assolement a évolué vers un système de monoculture où cette denrée se cultive toute l'année ou soit plusieurs années sur les mêmes parcelles. Ce qui n'est pas sans conséquences notamment sur le plan de fertilité du sol et phytosanitaire (Bardy, 2000). Dans un tel type d'exploitation, la baisse du niveau de fertilité de ce sol, s'exprime à son tour par une diminution progressive ou graduelle de rendement selon le type de sol (Bekunda et al., 2010). Mokuba et al. (2013) affirment que la culture continue du maïs entrâne une balance négative en matière organique $\mathrm{du}$ sol (MOS). Or, sans la restitution de ces MOS, le sol accuse une perte progressive de sa fertilité (Sanchez et Jama, 2002 ; Gichuru et al., 2003; Muyayabantu et al., 2012b).

Généralement, l'agriculture moderne est essentiellement dépendante d'intrants inorganiques pour une production intensive (FAO, 2003 ; Bouet et al., 2012; Tunya et al., 2014). Mais l'usage de ces fertilisants inorganiques comme source de nutriment aux cultures engendre non seulement le surplus de production mais aussi d'autres problèmes liés à la santé de l'homme et à la dégradation de l'environnement (Jeptoo et al., 2013). Il s'ajoute en plus le problème des coûts des ces engrais chimiques qui dans la plupart de cas sont exorbitants pour la plupart de petits producteurs des zones rurales.

$\mathrm{Vu}$ sous cet angle, les pratiques non chimiques d'amélioration de la fertilité du sol constituent un challenge de sortie par rapport aux méthodes classiques de fertilisation des sols par les engrais chimiques. Les causes majeures de fertilité faible de ces sols sont nombreuses dont on peut citer: les larges extensions des roches siliceuses, les érosions mécaniques, l'action dissolvante de l'eau, les phénomènes d'altérations chimiques, la disparition rapide de la matière organique du sol, etc. (Michel, 2006) dont la conséquence directe est le bas rendement dans l'agriculture de subsistance. Ces phénomènes sont renforcés en milieux ruraux africains par le raccourcissement du temps de repos accordé à la terre - période de jachère - voire, en maintes régions, supprimé (Michel, 2006) suite à la forte demande de la terre par les exploitants, conséquence d'une explosion démographique de la population ce dernier siècle. D'où, il est donc essentiel d'accorder une attention particulière à ce phénomène de perte de fertilité de sol. Mukendi et Ngoie (2015) pensent que rétablir les potentiels productifs des sols passe par la reconstitution de ses propriétés physiques, biologiques et chimiques.

Les recherches récentes mettent l'accent sur l'agriculture biologique pour répondre à ces triples défis d'ordre : physiques, chimiques et biologiques du sol. Elle s'appuie sur plusieurs technologies dont la fertilisation organique et le compost. Ces 
pratiques apportent significativement des quantités en éléments nutritifs au sol pour les cultures et ont un effet à long terme sur les propriétés du sol. Venkateswarlu et al. (2008) avaient documenté l'augmentation de 10.6\% le rendement de soja et du blé dur trois ans après application des fertilisants organiques au sol comparativement à la fumure inorganique seule. Les engrais organiques et les compost augmentent le carbone organique, l'activité biologique, l'humidité du sol et aussi l'azote, le phosphore, le potassium, le magnésium et la calcium (Venkateswarlu et al., 2008 ; Jeptoo et al., 2013; Chepkemoi., 2014;).

Cependant, malgré l'intérêt grandissant de l'application des engrais organiques en agriculture dans le monde, très peu d'attention a été accordée à ces technologies en milieu paysan de la zone d'étude. Alors que certaines espèces réputées fertilisantes sont présentes dans la zone telles que: Tithonia diversifolia, Sena floribunda et Entada abyssinica. Il y a donc besoin d'évaluer ces espèces en vue de diversifier les options disponibles et de réduire la dépendance des planteurs vis-àvis des engrais inorganiques dans la zone d'étude. Ainsi, nous supposons que l'application au sol des biomasses de $T$. diversifolia $S$. floribunda et E. abyssinica, qui sont des espèces à biomasses facilement décomposables et riches en éléments nutritifs (Kaho et al., 2011) accroîtraient le rendement du maïs var. Salongo II par rapport à l'apport minéral de DAP.

\section{MATERIEL ET METHODES}

\section{Milieu d'étude}

L'étude a été conduite au jardin expérimental de l'Université Notre Dame de la Lomami situé à Kimulo, dans le secteur de Kabinda, localité de Kananga à environs 16 $\mathrm{km}$ du côté Est de la cité Kabinda., territoire de Kabinda, Province de Lomami en République Démocratique du Congo. Les coordonnés géographiques du site expérimental sont le suivantes $6^{\circ} 06^{\prime} \mathrm{S}$, 243' ' E à $792 \mathrm{~m}$ d'altitude. La distribution annuelle de pluie est bimodale avec de pic en janvier et avril. La pluviométrie annuelle varie entre 1400 à $1600 \mathrm{~mm}$ de pluie et la température moyenne annuelle est d'environ $24{ }^{\circ} \mathrm{C}$. Les sols sont généralement acides, appartement au groupe de ferralsols d'après la classification de l'INEAC de 1961 (Ministère du plan, 2005). Le site a servi auparavant comme une jachère naturelle dont la végétation dominante était constituée d'Impérata cylindrica, Hypparhenia sp, Ptéridium aquilum, Entada abysinica, Panicum repens, Paspalun willee, Sorghum hapalens etc.

\section{Matériel}

Le matériel végétal était constitué d'une part d'une variété améliorée de maïs Salongo 2, obtenue à la station de recherche de Gandajika. Cette variété est inscrite au catalogue national des variétés homologuées par le service National de Semence (SENASEM), et d'autre part, par des biomasses d'E. abyssinica, de S. floribunda et de $T$. diversifolia. En fin, l'engrais composé (DAP) (16-46-0) avait servi de fertilisant minéral.

\section{Méthodes}

L'essai a été conduit pendant deux saisons culturales. La saison A a débuté en septembre 2013 et la saison B en février 2014. La préparation du terrain s'est réalisée du 10 au 20 septembre 2013 soit dix jours avant l'enfouissement des engrais verts pour la saison culturale A, et du 04 au 10 janvier 2014 
soit six jours avant l'enfouissement des engrais vert en saison B.

Les essais ont été conduits suivant un dispositif en blocs aléatoires complètements randomisés avec 4 répétitions. Les traitements mis à contribution étaient composés d'un témoin ou sans apport (T0), d'un apport d'E. abyssinica (T1), de $T$. diversifolia (T2), de $S$. floribunda (T3), d'une application au sol de la fumure minérale à base de DAP (T4). Les biomasses utilisées comme engrais vert ont été enfouies dans les 15 premiers centimètres du sol 10 jours avant le semis du maïs pour chaque période de plantation. L'enfouissement était localisé dans les sillons de semis. Le poids de biomasse reçu pour chaque parcelle correspond à 8t/ha. La parcelle élémentaire dans l'expérience était de $8 \mathrm{~m}^{2}$.

Le DAP avait été localisé dans le trou de semis du maïs à raison de $80 \mathrm{~kg} / \mathrm{ha}$. Le maïs a été semé aux écartements de $0.75 \mathrm{~cm}$ x 0.5 $\mathrm{cm}$ à raison de deux grains par poquet. Les soins d'entretiens se résument par le sarclage et le buttage.

Cette expérimentation a été appréciée avec les paramètres de croissance et de production de la culture. Les paramètres de croissances ont été mesurés pendant la phase végétative. Les caractères observés à la période végétative concerne la hauteur des plants, le diamètre au collet et le nombre des feuilles à la période d'initiation florale du maïs tandis que ceux de productions ont été mesurés à la récolte. Il a s'agit de la longueur et le diamètre des épis, le nombre de rangée par épi, le poids de 100 grains et le rendement à l'hectare après avoir séchés et pesées afin d'établir des moyennes correspondantes à chaque variable pour chaque traitement et chaque répétition.

\section{Analyse statistique}

Les données collectées ont été soumises à l'analyse de variances (ANOVA) à l'aide du logiciel Statistix 8.0 et les moyennes ont été séparées en utilisant le test de la plus petite différence significative (PPDS) au seuil de 0,05 de probabilité.

\section{RESULTATS}

L'évolution de la croissance des plants de maïs 60 jours après semis a pris en compte la hauteur, le diamètre au collet et le nombre des feuilles à chaque saison culturale au jardin expérimental de l'Université Notre Dame de Lomami. Ces résultats sont décrits au Tableau 1.

Les résultats obtenus en ce qui concerne la hauteur des plant durant le deux saisons culturales ont permis d'indiquer qu'ils existent des différences significatives en ce qui concerne la croissance en hauteur du maïs d'après l'analyse de la variance (Tableau 1). Les moyennes de la variable hauteur des plants montre que le témoin a une taille la plus faible du groupe dans chaque saison soit 101.95 et $85.85 \mathrm{~cm}$ respectivement pour la saison A et B, alors que les biomasses enfouies de $S$. floribunda ont accusées une hauteur de plant supérieure en saison A (154.6 $\mathrm{cm}$ ) alors qu'en saison B, c'est E. abyssinica qui a pris la tête avec $128.4 \mathrm{~cm}$ du groupe.

De même, pour le nombre de feuille à 60 jours de végétation, la même tendance se confirme à la saison $\mathrm{A}, S$. floribunda est en tête avec 14.67 feuilles contre 12 feuilles pour la parcelle témoin dans l'expérience, alors qu'en saison B aucune différence significative n'a été décelée entre les traitements dans l'expérience (Tableau 1).

En plus, le diamètre au collet du maïs soumis aux amendements organiques montre 
l'existence d'une différence significative (Tableau 1). La valeur la plus élevée du diamètre au collet a été celle de S. floribunda d'une part $(1.95 \mathrm{~cm})$ à la saison $\mathrm{A}$ et d'autre part avec E. Abyssinica $(2.10 \mathrm{~cm})$ en saison B contre le diamètre plus faible avec le témoin qui a enregistré $1.37 \mathrm{~cm}$ respectivement pour la saison A et B (Tableau 1).

Les paramètres de production ont été observés et mesurés après récolte du maïs en champ. Les résultats sont résumés dans le Tableau 2.

La longueur des épis récolté à la saison A varie, en moyenne, entre 8.05 et $9.25 \mathrm{~cm}$ (Tableau 2). L'analyse de la variance indique qu'il n'existe pas de différence significative. Sinon, la saison B montre que les parcelles ayant reçues des apports organiques que minéraux en ce qui concerne la longueur des épis après récolte, démontrent que tous les traitements accusent une supériorité statistiquement significative sur le témoin (Tableau 2), lequel a présenté les épis les plus courts possible $(10.47 \mathrm{~cm})$ par rapport aux DAP qui a enregistré la haute valeur du groupe soit $13.85 \mathrm{~cm}$.

Concernant le diamètre au milieu des épis, il découle des résultats consignés dans le Tableau 2 qu'au cours de la saison A, aucune différence statistiquement significative n'a été observée entre tous les traitements dans l'expérience, mais en saison $\mathrm{B}$, l'application de DAP prend la tête du groupe avec $4.55 \mathrm{~cm}$ alors que le témoin présente un diamètre plus faible $(4.10 \mathrm{~cm})$ comme le montre au Tableau 2.

Quant au nombre de rangées par épi, les moyennes enregistrées pour chaque traitement ne décèle aucune différence au regard de l'ANOVA pendant les deux saisons de cultures.
Le poids de cent grains de maïs sous l'effet des fertilisants à la saison A comme consigné dans le Tableau 2, présente des moyennes statistiquement significatives. DAP est supérieur du groupe avec 30 gr par contre le témoin avec le poids le plus faible avec 23.25 gr. Cependant, en saison B, aucune différence significative n'a été observée entre les traitements sous épreuves. Le rendement net du maïs observé durant la saison $\mathrm{A}$ et $\mathrm{B}$ sous l'effet des fertilisants est présenté à la Figure 1.

Il ressort de cette Figure 1 que l'apport au sol des fumures organiques et minérales a donné des moyennes de rendement significativement supérieures comparativement au témoin pendant les deux saisons de culture. Ces amendements organiques et minéral ont agit positivement sur le rendement du maïs. Le gain en poids enregistré sur chaque matière incorporée prouve à suffisance que chaque fertilisant apporté a joué un rôle additif rendant disponible les éléments minéraux essentiels dans le sol pour soutenir la production. En saison $\mathrm{A}$, le rendement supérieur du groupe a été observé avec le traitement à base de $T$. diversifolia $(1398.1 \mathrm{~kg} / \mathrm{ha})$ contre le témoin qui a enregistré un rendement faible du groupe soit $885.5 \mathrm{~kg} / \mathrm{ha}$. Comparativement à la saison $\mathrm{B}$, il s'est observé que le traitement minéral à base de DAP a pris le dessus du groupe (3332.8 kg/ha) alors que le témoin a le plus faible rendement de tous les traitements (1825.kg/ha) Figure 1. 
Tableau 1 : Evolution végétative du maïs à Kimulo saison A et B.

\begin{tabular}{|c|c|c|c|c|c|c|}
\hline \multirow[t]{3}{*}{ Traitements } & \multicolumn{6}{|c|}{ Evolution végétative à la floraison } \\
\hline & \multicolumn{2}{|c|}{ Hauteur (cm) } & \multicolumn{2}{|c|}{ Nombre de feuilles } & \multicolumn{2}{|c|}{ Diamètre au collet $(\mathrm{cm})$} \\
\hline & Saison A & Saison B & Saison A & Saison B & Saison A & Saison B \\
\hline T0 & $101.95 \mathrm{~b} \pm 0.57$ & $85.85 \mathrm{c} \pm 2.39$ & $12.00 \mathrm{~b} \pm 0.20$ & $9.20 \mathrm{a} \pm 0.24$ & $1.37 \mathrm{~b} \pm 0.11$ & $1.37 \mathrm{c} \pm 0.11$ \\
\hline $\mathrm{T} 1$ & $136.68 \mathrm{a} \pm 2.91$ & $128.40 \mathrm{~b} \pm 1.85$ & $14.42 \mathrm{a} \pm 0.29$ & $10.10 \mathrm{a} \pm 0.47$ & $1.77 \mathrm{ab} \pm 0.08$ & $2.10 \mathrm{a} \pm 0.04$ \\
\hline $\mathrm{T} 2$ & $134.60 \mathrm{a} \pm 2.36$ & $120.83 a \pm 0.61$ & $13.95 \mathrm{a} \pm 0.32$ & $10.15 \mathrm{a} \pm 0.12$ & $1.65 \mathrm{ab} \pm 0.05$ & $1.67 b \pm 0.11$ \\
\hline $\mathrm{T} 3$ & $154.60 \mathrm{a} \pm 4.40$ & $127.68 \mathrm{a} \pm 0.48$ & $14.67 \mathrm{a} \pm 0.36$ & $10.12 \mathrm{a} \pm 0.30$ & $1.95 \mathrm{a} \pm 0.08$ & $1.82 \mathrm{ab} \pm 0.08$ \\
\hline $\mathrm{T} 4$ & $134.45 \mathrm{a} \pm 7.02$ & $110.48 b \pm 2.53$ & $13.82 \mathrm{a} \pm 0.74$ & $9.67 a \pm 047$ & $1.80 \mathrm{ab} \pm 0.12$ & $1.90 \mathrm{ab} \pm 0.02$ \\
\hline $\mathrm{CV}(\%)$ & 6.77 & 2.96 & 5.31 & 6.20 & 10.76 & 9.3 \\
\hline PPDS (5\%) & 13.71 & 5.18 & 1.11 & N.S & 0.28 & 0.39 \\
\hline
\end{tabular}

$\mathrm{CV}$ : coefficient de variation ;PPDS : plus petite différence significative ; N.S : non significatif ; T0 : Témoin ; T1 : E. abyssinica, T2 : T. diversifolia ; T3 : S. floribunda ; T4 : DAP 
Tableau 2 : Paramètres de production du maïs en saison A 2013 et B 2014.

\begin{tabular}{|c|c|c|c|c|c|c|c|c|}
\hline \multirow[t]{2}{*}{ Traitements } & \multicolumn{2}{|c|}{ Longueur épi (cm) } & \multicolumn{2}{|c|}{ Diamètre épi (cm) } & \multicolumn{2}{|c|}{ Nbre Rangé par épi } & \multicolumn{2}{|c|}{ Poids 100 graines } \\
\hline & Saison A & Saison B & Saison A & Saison B & Saison A & Saison B & Saison A & Saison B \\
\hline T0 & $9.07 \mathrm{a} \pm 0.41$ & $10.47 \mathrm{~b} \pm 0.70$ & $3.80 \mathrm{a} \pm 0.23$ & $4.10 c \pm 0.18$ & $12.75 a \pm 0.60$ & $13.65 a \pm 0.54$ & $23.25 \mathrm{c} \pm 1.65$ & $23.75 \mathrm{a} \pm 1.43$ \\
\hline $\mathrm{T} 1$ & $9.25 \mathrm{a} \pm 0.33$ & $13.00 \mathrm{a} \pm 0.089$ & $4.00 \mathrm{a} \pm 0.05$ & $4.32 \mathrm{ab} \pm 0.10$ & $13.52 \mathrm{a} \pm 0.16$ & $14.37 \mathrm{a} \pm 0.34$ & $28.25 \mathrm{ab} \pm 0.62$ & $25.75 a \pm 085$ \\
\hline $\mathrm{T} 2$ & $8.90 \mathrm{a} \pm 0.48$ & $12.55 \mathrm{ab} \pm 0.55$ & $3.67 \mathrm{a} \pm 0.23$ & $4.47 \mathrm{a} \pm 0.17$ & $13.67 \mathrm{a} \pm 0.18$ & $13.75 a \pm 0.52$ & $23.75 b c \pm 0.62$ & $26.75 \mathrm{a} \pm 1.31$ \\
\hline $\mathrm{T} 3$ & $9.05 \mathrm{a} \pm 0.82$ & $12.90 \mathrm{ab} \pm 0.43$ & $3.77 \mathrm{a} \pm 0.21$ & $4.37 \mathrm{ab} \pm 0.08$ & $12.85 \mathrm{a} \pm 0.39$ & $13.95 \mathrm{a} \pm 0.08$ & $25.25 b c \pm 0.85$ & $25.75 a \pm 0.85$ \\
\hline $\mathrm{T} 4$ & $8.05 a \pm 0.62$ & $13.85 \mathrm{a} \pm 0.47$ & $3.82 \mathrm{a} \pm 0.22$ & $4.55 \mathrm{a} \pm 0.10$ & $12.35 \mathrm{a} \pm 0.65$ & $14.10 \mathrm{a} \pm 0.24$ & $30.00 \mathrm{a} \pm 0.70$ & $25.25 \mathrm{a} \pm 1.25$ \\
\hline CV $(\%)$ & 8.4 & 8.4 & 10.8 & 4.8 & 6.5 & 4.21 & 7.6 & 9.17 \\
\hline PPDS (5\%) & N.S & 1.6 & N.S & 0.3 & N.S & N.S & 2.69 & N.S \\
\hline
\end{tabular}




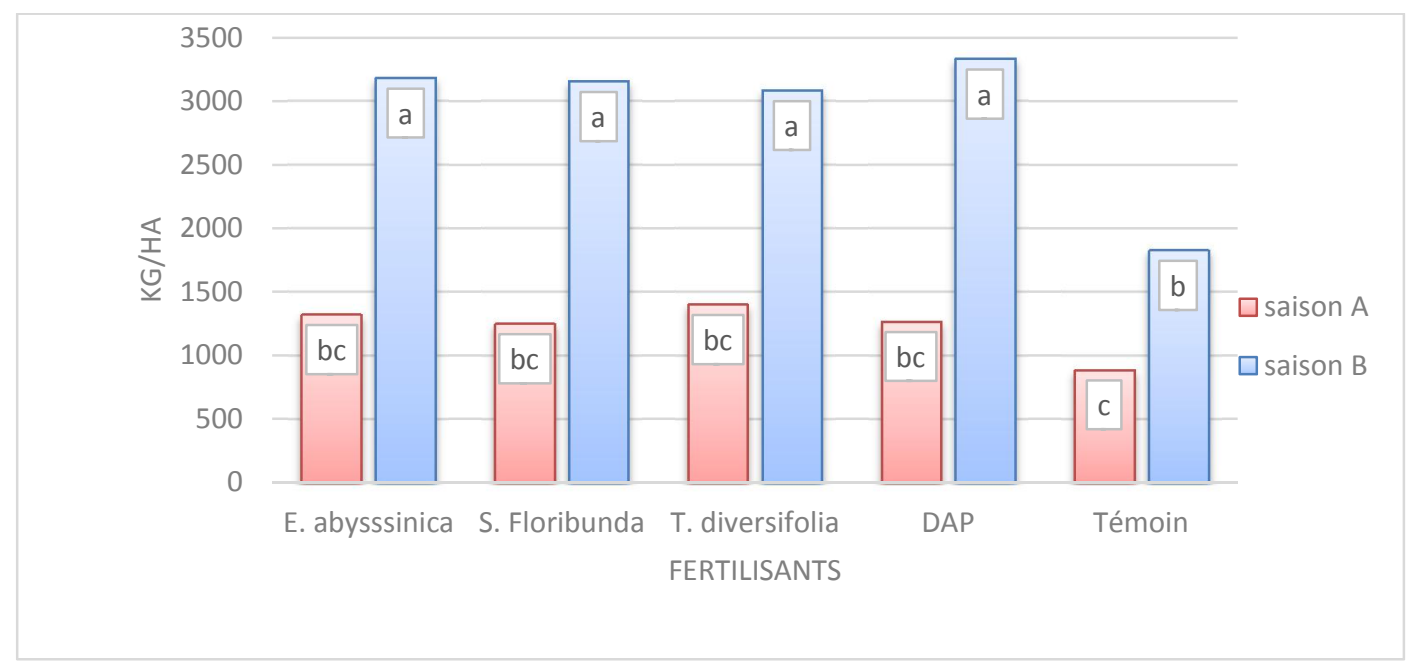

Figure 1 : Rendement du maïs par rapport aux saisons culturales.

\section{DISCUSSION}

En réponse du niveau de fertilisant utilisé dans cette étude, la tendance générale des paramètres de croissances observer dans le Tableau 1 démontre que les parcelles témoins ont présenté les plants de maïs avec une faible vigueur de croissance tant en hauteur, diamètre au collet qu'en nombre de feuille par rapport aux plants situés dans les parcelles ayant reçu les fertilisants organiques que minéral quelle que soit la saison culturale. Ceci serait probablement dû à la disponibilité des nutriments par les fertilisants utilisés. Ces observations corroborent celles de Adekayode et Ogunkoya (2011). Le sol où l'étude a été placée nous semble de toute évidence avoir la fertilité native basse en $\mathrm{N}$, pourtant c'est l'élément majeur indispensable pour la croissance des végétaux. Cela pourrait être la cause de la réduction de la performance de la variété du maïs salongo2 comme l'a décrit SENASEM (2008). Cependant, l'apport au sol des fertilisants organiques et minéral, a stimulé la croissance végétative des plants de maïs et a eu des effets sur le rendement. Ainsi, nos observations rejoignent celles de Olabode et al. (2007), Muyayabantu et al. (2012a et b),
Moke et al. (2013), et Namoi et al. (2014). En plus, les valeurs moyennes des paramètres de productions, dont la longueur, diamètre, nombre de rangées de l'épi et poids de cent grains observés sous de divers traitement organiques et inorganique, sont lié d'une part à la propriété intrinsèque de ladite variété et d'autre part aux propriétés du sol sous étude. Les moyennes de rendements en grains de maïs obtenues suivant la source et la nature de la matière fertilisante apportée au sol dans la présente expérimentation en saison $\mathrm{A}$ qu'en saison B ont été significativement supérieures par rapport au témoin et ont varié entre $885 \mathrm{~kg}$ et $1398.1 \mathrm{~kg}$ à la saison $\mathrm{A}$ contre $1825 \mathrm{~kg}$ et $3332.8 \mathrm{~kg}$ en saison $\mathrm{B}$ respectivement pour le témoin et les matières fertilisantes utilisée dans cette étude, engendrant simultanément plus de $63.3 \%$ de rendement en saison $\mathrm{A}$ et $54,75 \%$ en saison $\mathrm{B}$ suite à l'utilisation des fertilisants indistinctement de leur nature. Le rendement de maïs sous application des fertilisants organiques au jardin expérimental de l'Université Notre Dame donne des résultats similaires à ceux obtenus en station de recherche, fixé à 4 t/ha (SENASEM, 2008). Orwa et al. (2009) ont indiqué que de bon 
rendement de la culture du maïs sont obtenu avec application de $5 \mathrm{t} / \mathrm{ha}$ de biomasse de $T$. diversifolia. Dela Pena et al. (2013) ont obtenu des performances de croissance et de production avec le $T$. diversifolia sur le chou. Ces résultats de la Figure 1 illustrent le pouvoir fertilisant de $T$. diversifolia, de E.abyssinica, de S. floribunda comme l'ont décrit (Orwa et al., 2009; Muyayabantu, 2010 ; Jeptoo et al., 2013) dans leurs études. Le niveau de fertilité du sol atteint, suite aux apports des biomasses végétales est en corrélation avec le rendement du maïs. C'est surtout le caractère intrinsèque des la composition chimique de biomasses sous étude. Savini et al. (2006), affirment que le processus de décomposition de $T$. diversifolia conduit à la libération du $\mathrm{C}$ sous forme de $\mathrm{CO}_{2}$ dans l'atmosphère, la conversion de $\mathrm{N}$ organique sous la forme $\mathrm{NO}_{3}$ et $\mathrm{NH}_{4}{ }^{+}$par le processus biologique d'ammonification et de nitrification respectivement deux semaines après leur application. Cette corrélation justifierait les résultats relatifs au rendement du maïs observé à la Figure 3. Ces résultats rejoignent l'observation par Muyayabantu et al. (2012a et b) que l'application de 8 tonnes de E.abyssinica et de $T$. diversifolia avait augmenté significativement la production de maïs à Gandajika jusqu' à plus de 4 t/ha par rapport aux parcelles témoin.

\section{Conclusion}

En système traditionnel, la pratique du champ sur brulis raréfie les terres agricoles et les paysans préconisent d'ordinaire l'assolement qui dépend étroitement de la nature du sol. Dans un tel type d'exploitation, la baisse du niveau de fertilité de ce sol s'exprime à son tour par une diminution progressive ou graduelle de rendement suite a une balance négative en matière organiques du sol, une perte de progressive de sa fertilité. De cette étude, il est évident que le rendement de la culture du maïs ne peut pas être amélioré de façon durable au cours du temps sans l'amélioration de la fertilité du sol. Il s'est observé que le maïs, sous différents apports aussi bien organiques que minéral, a présenté une bonne végétation en phase de croissance par rapport aux parcelles non traitées. L'apport des fertilisants a accéléré la croissance végétative, une augmentation de l'ordre de 40 à $80 \%$ du rendement en grain. Ces résultats peuvent constituer un guide d'utilisation de de E.abyssinica, T. diversifolia de $S$. floribundadans par des pratiques diverses, surtout pour tout agriculteur de cette zone.

\section{CONFLIT D'INTERETS}

Les auteurs déclarent qu'aucun conflit d'intérêt n'existe par rapport à cet article.

\section{CONTRIBUTIONS DES AUTEURS}

Les auteurs ont participé de diverses manières à la conception de cet article, RTM est l'investigateur principal. Il a défini les protocoles et fait les analyses statistiques; BTM et OMK ont apporté un appui technique et ont participé à la rédaction du manuscrit, TMM et LBL ont corrigé le manuscrit ; dirigé et managé le travail.

\section{REFERENCES}

Adekayode FO, Ogunkoya MO. 2011. Comparative effects of organic compost and NPK fertilizer on soil fertility, yield and quality of amaranth in southwest Nigeria. Int. J. Biol. Chem. Sci., 5(2): 490-499.

Bardy C. 2000. La culture du maïs en République démocratique du Congo. In Agrinfo, dossier spécial maïs, éd. Midema ; 6-7.

Bationo A, Hartemink A, Lungu O, Naimi M, Okoth P, Smaling E, Thiombiano L. 2006. African soils: their productivity and profitability of fertilizer use, In: Proceedings of the African Fertilizer Summit. June 9-13, 2006, Abuja, Nigeria, 29. 
Bekunda B, Sanginga N, Woomer PL. 2010. Restoring Soil Fertility in Sub-Sahara Africa. Advances in Agronomy, 108: 184-236. DOI: 101016/S0065-2113(10)

Bouet A, Nicaise Acho Amancho A, Sanogo S, Camara M. 2012. Effet de la fertilisation azotée et phosphorée sur le développement de la Panachure jaune en riziculture aquatique en Côte d'Ivoire. Int. J. Biol. Chem. Sci., 6(6): 4071-4079. DOI: $\quad$ ttp://dx.doi.org/10.4314/ ijbcs.v6i6.17

Chepkemoi J, Onwonga RN, Karuku GN, Kathumo VM. 2014. Effect of tillage practices, cropping systems and organic inputs on soil moisture cotent in Yatta sub-contry, Kenya, In innovative research and technology for global development. Proceedings 26-28 mars, Egerton university Press, 274-279.

Dela Pena, Cenny I, Bartolome DL. 2013. The potential of Tithonia diversifolia (Wild Sunflower) as organic foliar fertilizer. European Scientific Journal, 4: 465-468.

FAO. 2003. Association Internationale de l'Industrie des Engrais, Les engrais et leurs applications: Institut Mondial du Phosphate : Rabat ; 76.

Gichuru MP, Bationo A, Bekunda MA, Goma HC, Mafongonya PL, Mugendi DN, Murwira HM, Nandwa SM, Nyathi P, Swift MJ. 2003. Soil Fertility Management in Africa: A regional Perspective. Academy Science publishers (ASP) in association with the tropical Soil Biology and Fertility of CIAT: Nairobi; 306.

Jeptoo A, Aguyoh JN, Saidi M. 2013. Tithonia manure improves carrot yield and quality. G.J.B.A.H.S., 2(4): 136-142.

Kaho F, Yemefack M, Feujio-Teguefouet P, Tchantchaouang JC. 2011. Effet combiné des feuilles de Tithonia diversifolia et des engrais inorganiques sur les rendements du maïs et les propriétés d'un sol ferralitique au Centre Cameroun. Tropicultura, 29(1): 39 - 45.

Michel M. 2006. Développement Intégré des régions tropicales, Tome I, Bases biophysiques de l'environnement tropical.

Ministère du Plan. 2005. Monographie du Kasaï Oriental. République Démocratique du Congo, 149p.

Mokuba W, Kizungu RV, Lumpungu K. 2013. Evaluation de l'effet fertilisant de Mucuna utilis L. face à deux doses de NPK (17-17-17) sur la croissance et la production de la variété samaru du maïs (Zea mays L.) dans les conditions optimales. Congo Science, 1(1): 24-27.

Nkoy Moke KN, Ayingwe L, Ndiku LL, Babelangi A. 2013. Amendement des sols : Influence des fertilisants pour l'amélioration de la culture de Vigna unguiculata (L) Walp. Int. J. Biol. Chem. Sci., 7(5): 2029-2039. DOI: http://dx.doi.org/10.4314/ijbcs.v7i5.20

Mukendi TR, Ngoie KJ. 2015. Effet des engrais biologiques sur la production légumière d'amarante (Amaranthus hybridus). Cahiers du Cresa. 179-193.

Mukendi TR, Tshilumba MT, Mpoyi BM, Mutamba NB, Kabongo MD, Ilunga TM, Ngoie KJ, Ngoyi ND, Munyuli MT. 2017. Évaluation de la productivité du maïs (Zea mays L.) sous amendements organique et minéral dans la province de Lomami, République Démocratique du Congo. Journal of Applied Biosciences 109: 10571-10579. DOI: http://dx.doi.org/10.4314/jab.v109i1.2

Muyayabantu GM, Kadiata BD, Nkongolo KK. 2012b. Response of maize to different organic and inorganic fertilization regimes in monocrop and intercrop systems in a sub-Saharan Africa region. Journal of Soil Science and Environmental Management, 3(2): 42-48. DOI: 10.5897/JSSEM11.079. 
Muyayabantu GM, Kadiata BD, Nkongolo KK. 2012a. Evaluation of Biological Soil Fertility Management Practices for Corn Production in Oxisols. American Journal of Plant Sciences, 3:1654-1660. DOI: doi:10.4236/ajps.2012.311201

Muyayabantu GM. 2010. Gestion du système cultural et de fertilité biologique du sol en vue de la production du maïs dans la contrée de Gandajika. In Recherches Agronomiques en Milieu Paysan pour une Sécurité Alimentaire Durable en RD-Congo, Nkongolo KK, Kizungu V, Kalonji-Mbuyi A (eds). Sudbury: Ontario, Canada ; 105-160.

Namoi NL, Onwonga RN, Karuku GN, Kathumo VM, Onyango CM. 2014. Assessment of soil nutrient flows and balances in organic based cassava (Manihot esculenta Crantz) and sorghum (Sorghum bicolor (L.) Moench) cropping systems of Kitui subcounty, Kenya. In innovative research and technology for global development. Proceedings 26-28 mars. Egerton University Press; 185-193.

Ngome AF, Mtei KM, Tat PI. 2012. Mucuna pruriens differentially affect maize yields in three soils of Kakamega District. Int. J. Biol. Chem. Sci., 6(3): 941-949. DOI: http://dx.doi.org/10.4314/ijbcs.v6i3.3

Olabode OS, Ogunyemi S, Akanbi WB, Adesina GO, PA Babajide. 2007. Evaluation of Tithonia diversifolia (Hemsl.) A Gray for Soil Improvement, World Journal of Agricultural Sciences, 3(4): 503-507.

Orwa C, Mutua A, Kindt R, Jamnadass R, Simons A. 2009. Agroforestree Database:a tree reference and selection guide version 4.0 (http://www.worldagroforestry.org/af/tre $\mathrm{edb} /$ ).
Sanchez PA, Jama BA. 2002. Soil fertility replenishment takes off East and Southern Africa. In Integrated Plant Nutrition Management in Sub-Saharan Africa from Concept to Practice, Vanlauwe B (ed). CABI International; 23-45.

Savini I, Paul C, Smithson KN, Karan, Yamasaki H. 2006. Influence of Tithonia diversifolia and triple superphosphate on dissolution and effectiveness of phosphate rock in acidic soil. J. Plant Nutr. Soil Sci., 5(169): 593-604. DOI : 101002/jplr.200521931

SENASEM. 2008. Catalogue variétale des cultures vivrières, maïs, riz, haricot, arachide, soja, niébé, manioc, patate douce, pomme de terre, banane. CTB-M, Kinshasa, 148p.

Tahir M, Javed MR, Tanveer A, Rehman JU. 2009. Effect of different herbicides on weeds, growth and yield of spring planted maize (Zea mays L.). Pak. J. Life Soc. Sci., 7(2): 168-174.

Tunya BA, Lelei JJ, Ouma JP. 2014. Effect of chicpea (Cicerarietinum L.) and white lupin (Lupinusalbus L.) on phosphorus mobilization from Minjingu phosphate rock, soil chemical properties and plant nutrient uptake in various cropping systems. In innovative research and technology for global development. Proceedings 26-28 mars. Egerton University Press; 301-315.

Venkateswarlu B, Balloli SS, Ramakrishna YS. 2008. Organic Farming in Rainfed Agriculture: Opportunities and Constraints. Central Research Institute for Dryland Agriculture: Hyderabad; $185 \mathrm{p}$. 